\title{
A Statistical Comparative Study of Photoplethysmographic Signals in Wrist- Worn and Fingertip Pulse-Oximetry Devices
}

\author{
Kais Gadhoumi ${ }^{12}$, Kevin Keenan ${ }^{3}$, Rene Colorado ${ }^{3}$, Karl Meisel $^{3}$, Xiao $\mathrm{Hu}^{12}$ \\ ${ }^{1}$ Department of Physiological Nursing, School of Nursing, UCSF, San Francisco, California, USA \\ ${ }^{2}$ Center for Physiologic Research, UCSF, San Francisco, California, USA \\ ${ }^{3}$ School of Medicine, UCSF, San Francisco, California, USA
}

\begin{abstract}
Prolonged time-matching continuous recordings of electrocardiogram and photoplethysmographic waveforms from fingertip pulse oximeter and wrist-worn devices obtained from stroke patients admitted to the intensive care unit were analyzed. Heart rate and pulse rate variability indices and signal complexity were estimated and statistically compared to investigate the reliability of heart rate estimation using photoplethymography and whether fingertip pulse oximetry and radial photoplethymography reflect the same pulse rate variability. Heart rates were generally found to be accurately estimated by both photoplethysmographic modalities when using the electrocardiogram as a reference. Pulse rate variability analysis showed statistically comparable properties between photoplethysmographic modalities, suggesting that fingertip pulse oximetry and wrist photoplethymography reflect the same cardiovascular changes.
\end{abstract}

\section{Introduction}

Photoplethymography (PPG) is an optical measurement technique used primarily in the detection of the blood volume changes and offers a convenient and cost-effective means for assessing vital signals like oxygen saturation, blood pressure, and cardiac output. The interest of using photoplethymography for monitoring health condition outside the hospital setting has recently increased with the abundance of wearable devices in the consumer market that offer readily available low-cost photoplethymography sensors powered with relatively long-lasting batteries.

Recent studies have shown that accurate detection of heart rhythm irregularities or arrhythmia using wearable devices is now possible [1]. However, in most of these studies, the analysis of the photoplethysmographic waveform was based on an intermittently recorded signal due to power saving requirements for a majority of existing consumer-grade wearable devices. In some patients with conditions of paroxysmal (intermittent) arrhythmia, noncontinuous analysis may lead to undetected episodes of arrhythmia resulting in underdiagnoses of otherwise actionable conditions such as atrial fibrillation, which is a major risk-factor that require careful extended screening [2]. Prolonged recording and continuous analysis of photoplethysmographic waveforms increase the chance of unraveling such risk-factors.

In this study, we analyzed continuous photoplethysmographic recordings from a wrist-worn device and a fingertip pulse oximeter device in a goal to assess their accuracy in estimating the heart rate and to evaluate whether photoplethymography of the radial artery reflect the same cardiovascular changes as those recorded in the peripheral capillaries of the hand finger.

\section{Methods}

\subsection{Patients and Data}

Fourteen patients (aged between 19 and 91; median=74) admitted in the intensive care units of the University of California San Francisco Medical Centre between October 2016 and January 2018 for ischemic stroke were prospectively selected to wear a wristband (E4 wristband, Empatica Inc, Cambridge, USA) for continuous recording of the radial blood volume pulse (BVP) while being monitored by standard bedside patient monitors. Patient consent and approval of the UCSF Institutional Review Board was obtained prior to patient enrollment. BVP waveforms were recorded with E4 wristband at $64 \mathrm{~Hz}$. Time-matching physiological waveform recordings of 7lead electrocardiogram (ECG) and fingertip pulse oximetry $(\mathrm{SpO} 2)$ acquired at $240 \mathrm{~Hz}$ were extracted for thirteen of fourteen patients (bedside monitor data was not accessible for one patient). Between 3 and $24.7 \mathrm{~h}$ of recordings were obtained (average duration $=13.5 \mathrm{~h}$ ). All analyses were carried out in Matlab (MathWorks, Inc).

\subsection{Signal Quality Assessment}


The BVP and $\mathrm{SpO} 2$ recordings were segmented into contiguous non-overlapping 30s-segments (simply referred to as segments in the rest of manuscript) for all patients and assessed for signal quality in each segment. The choice of the segment length was primarily dictated by the clinical definition of atrial fibrillation [3] given that this study subscribes under a larger study on atrial fibrillation. A singular value decomposition (SVD) based approach, adapted from Asgari et al. [4, 5], was used for the quality assessment procedure: A library of pulses is constructed for each segment and decomposed using SVD into signal and noise subspaces. Ratio of projected signal in the signal space to the projected signal in the noise space is calculated and compared to an empirically determined threshold. If the ratio is greater than the threshold, the segment is considered as a valid physiological signal $(\mathrm{QoS}=1)$, otherwise the signal is considered as a non-valid physiological signal (QoS = -1). Pulse onset was detected using two methods. The first method uses the weighted slope sum and adaptive thresholding approach for onset detection of blood pressure waveforms as proposed by Wong's et al [6]. The second method [7] implements a pulse onset detection algorithm for arterial blood pressure waveforms and it is largely inspired by the derivatives and thresholds described in PanTompkins method [8]. Both methods were visually assessed for correctly identifying the pulse onset in 50 randomly selected $\mathrm{BVP}$ and $\mathrm{SpO} 2$ recordings from all patients. The method with best accuracy was adopted for pulse detection in subsequent analyses.

To compare the trend of the quality of signal between $\mathrm{BVP}$ and $\mathrm{SpO} 2$ recordings for a given patients, the cumulative sum of the QoS values calculated over the segments was used. The spearman's rank correlation coefficient was then calculated for the QoS trends.

Only patients with 30 min or more of cumulated good quality $\mathrm{BVP}$ and $\mathrm{SpO} 2$ segments were selected for subsequent analyses for statistical power of results.

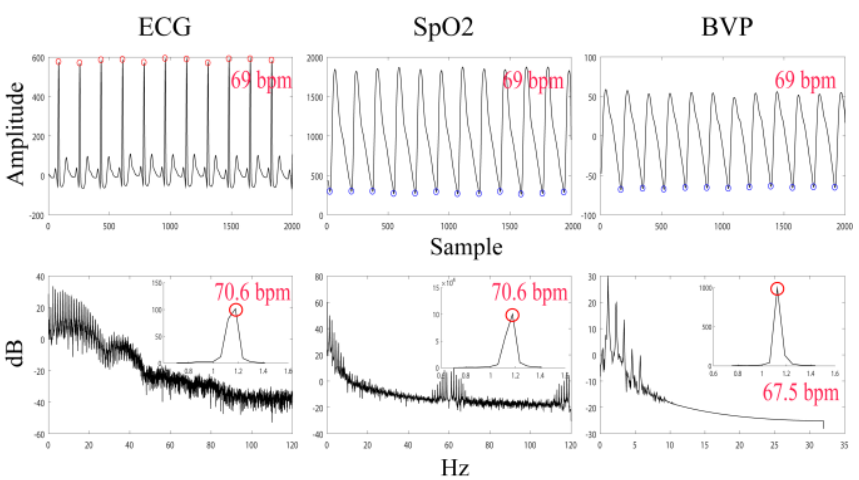

Figure 1. Illustration of temporal (upper panel) and spectral (lower panel) heart rate estimation.

\subsection{Pulse Rate Variability Analysis}

Inter-pulse intervals (IPIs) were derived from pulse onsets in $\mathrm{BVP}$ and $\mathrm{SpO} 2$ segments with good quality assessment by calculating the difference between successive pulse onsets.

For the calculation of the ECG heart rate, inter-beatintervals were extracted for each non-overlapping segment of ECG recordings by using a Pan-Tompkins algorithm [8] to detect the peaks of QRS complexes. Intervals corresponding to heart rates outside the physiological range of 30-240 bpm were considered outliers and were discarded.

The average heart rate and a series of time-domain and frequency-domain indices quantifying the amount of variability in the inter-pulse interval across each recording were calculated from the aggregated IPIs [9] and compared for statistical significance of the difference in mean values using two-sample Kolmogorov-Smirnov tests. Metric definitions are given in table 1.

Table 1 . Heart-rate and pulse rate variability metrics.

\begin{tabular}{cc}
\hline Index & Description \\
\hline HR & 60/Average IPI (bpm) \\
SDNN & Standard deviation of IPI (ms) \\
RMSSD & Root mean square of successive IPI (ms) \\
pNN50 & Percentage of successive IPI that differ \\
$(\%)$ & by more than 50 ms \\
pNN20 & Percentage of successive IPIs that differ \\
& by more than 50 ms $(\%)$ \\
Triangular & Integral of the density of IPIs histogram \\
Index & divided by its height (ms) \\
TINN & Baseline width of IPI histogram (ms) \\
Lf/Hf & Ratio of low ([0.04 0.15$\left.]_{\mathrm{Hz}}\right)$ to high \\
& $\left([0.150 .4]_{\mathrm{Hz}}\right)$ frequency power $(\%)$ \\
\hline
\end{tabular}

To compare signal complexities between recordings, the sample entropy [10] was used. Sample entropy values were calculated across each BVP and $\mathrm{SpO} 2$ recording (maximum template length $=2$, matching tolerance threshold $=0.2$ were used). Additionally, a frequencydomain analysis was carried out to compare the spectralbased estimation of the heart rate in ECG, SpO2 and BVP recordings. The power spectral density was estimated in each segment using Welch's method (2048 FFT points, $25 \%$ overlap ensured good spectral resolution). The heart rate was then estimated by finding the fundamental frequency of the power spectral density in the frequency band corresponding to the patient heart rate range as calculated in the HRV analysis. The fundamental frequency was determined by locating the first of equidistant peaks with significant spectral power. In most cases, the fundamental frequency was observed to be the peak frequency of the power spectrum. Fig. 1 shows an example of detected pulses and beats in time-matching 30 s-segments of BVP, $\mathrm{SpO} 2$ and ECG and the estimated heart rates using power spectral density analysis. 


\section{Results}

\subsection{Signal Quality Assessment}

Pulse detection was found to be more accurate with the second method than with the first method (See Methods) and was used in subsequent analysis. Five of thirteen patients enrolled in the study had $30 \mathrm{~min}$ or less of cumulative good quality segments and were not analyzed further. In all five patients, BVP recordings had very few segments (less than $2 \mathrm{~min}$ ) of good quality segments while $\mathrm{SpO} 2$ recordings in three of these five patients were totally of bad quality. Temporal trends of BVP and $\mathrm{SpO} 2$ signal quality were positively correlated in most patients (Fig. 3a). Spearman's $\rho$ was above 0.85 in eight of thirteen patients. In three of five patients where trends were poorly or negatively correlated, a high positive correlation could be appreciated in the first hours of recordings (patients $\mathrm{P} 2$, P8, and P9). Fig. 2b shows the proportion of good quality segments in each patient.

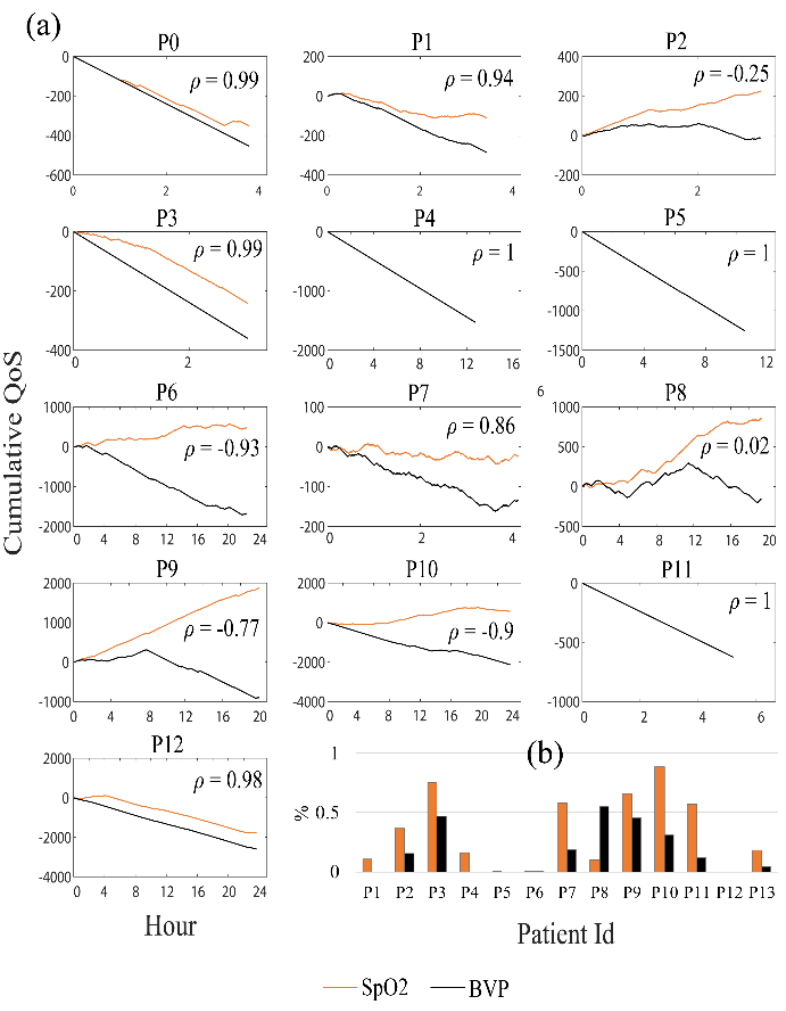

Figure 2. Trends of signal quality (a) and proportion of good quality segments (b).

\subsection{Heart Rate Estimation}

In eight of thirteen patients who had $30+$ min of good quality segments, the difference between inter-pulse interval values in $\mathrm{SpO} 2$ and BVP was not statistically significant $(p$-value $=0.93$; Two-sample Kolmogorov-
Smirnov test). The estimated average heart rate derived from the average inter-pulse interval in $\mathrm{SpO} 2$ and in BVP, and from the average inter-beat interval of ECG had very close values in each patient (Fig. 3 ; $p$-value $=0.79$; oneway ANOVA test).

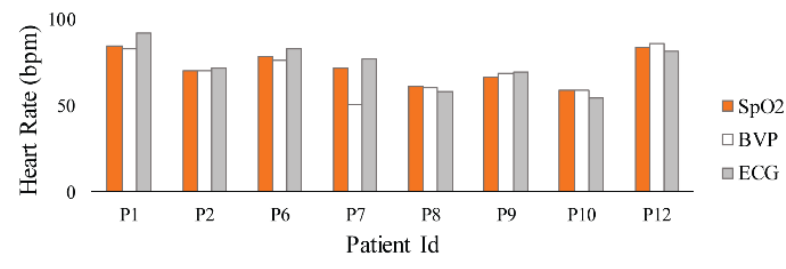

Figure 3. Average heart rates and inter-pulse intervals.

\subsection{Segment-Wise Heart Rates}

Heart rates estimated using power spectral density calculated in each BVP and SpO2 segment $\left(H R_{\{P S D\}}=\right.$ $60 * f$ with $f$ being the fundamental frequency of the signal in the physiological range) showed relatively poor agreement with segment-wise ECG heart rate values calculated using inter-beat intervals of each segment $(95 \%$ limits of agreement $= \pm 10 \mathrm{bpm}$ ). Heart rates estimated using IBIs showed however a good agreement with segment-wise ECG-based heart rate estimates. Fig. 4 illustrates such an agreement for eight of thirteen patients who had 30+ min of goof quality signals (spearman's $\rho=$ 0.97. $95 \%$ limits of agreement below the clinically acceptable difference threshold of $5 \mathrm{bpm})$.

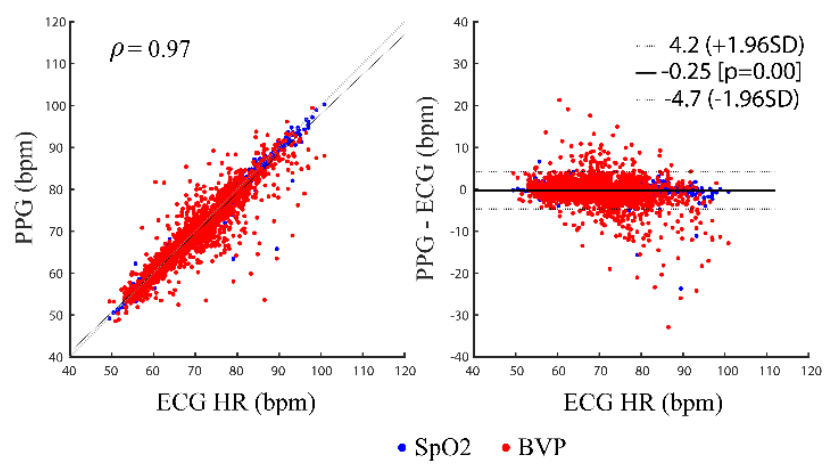

Figure 4. Correlation and agreement analysis (BlandAltman plot) between heart rates.

\subsection{Pulse Rate Variability and Signal Complexity Analysis}

Average values of the pulse rate variability indices and the sample entropy were comparable ( $p$-value $>0.05$; Twosample Kolmogorov-Smirnov test) among the eight patients who had $30+$ min of good quality signals (Fig. 5) in $\mathrm{BVP}$ and $\mathrm{SpO} 2$ recordings. 


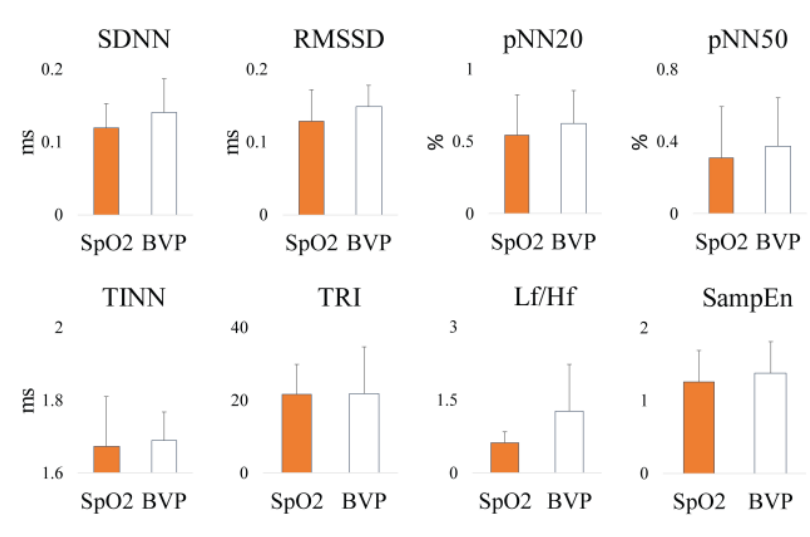

Figure 5. Average values of PRV indices and Sample Entropy.

\section{Conclusion}

A comparison between continuous and long-lasting photoplethysmographic and electrocardiographic recordings in a cohort of patients with ischemic stroke was presented. The findings are preliminary given the limited number of patients analyzed. However, results on the comparison between heart rates estimated using the electrocardiogram and photoplethysmograms corroborate early reports on the validity of the heart rate estimation in wearable devices under resting conditions [11]. Heart rate estimation under moderate or intense physical activity conditions remain controversial and was not a subject of this study [12,13]. To our knowledge, this study is the first to report a comparison between pulse-oximetry and photoplethymography from continuous prolonged recordings. In most patients, both modalities showed comparable statistical properties when analyzing the pulse rate variability. The quality of plethysmographic waveforms was also comparable between modalities for most of recording duration. The discrepancies of signal quality trends between both modalities in some recordings may be due to unsynchronized limb movement. In fact, in some patients, the E4 device and the fingertip pulse oximeter were worn on different hands. The statistical concordance between heart rates and PRV indices evaluated in both modalities suggest that $\mathrm{SpO} 2$ recordings can be used as surrogates for BVP recordings. Such interchangeability between signals recorded from different photoplethymography modalities can prove very useful in building large datasets of expertly annotated photoplethysmographic waveforms as a great deal of continuous $\mathrm{SpO} 2$ recordings is readily accessible in most healthcare institutions. Large annotated datasets can be extremely valuable in the training of data-hungry algorithms - such as in deep learning approaches - for the accurate detection of arrhythmia in wearable devices.

\section{References}

[1] J. M. Sanchez, B. Ballinger, J. E. Olgin, M. J. Pletcher, E. Vittinghoff, E. Lee, et al., "AF detection and ablation outcomes: answering questions that matter to patients: detecting atrial fibrillation using a smart watch - the mRhythm study," Heart Rythm Society, 2017.

[2] B. Freedman, J. Camm, H. Calkins, J. S. Healey, M. Rosenqvist, J. Wang, et al., "Screening for atrial fibrillation: a report of the AF-SCREEN international collaboration," Circulation, vol. 135, pp. 1851-1867, May 92017.

[3] P. Kirchhof, S. Benussi, D. Kotecha, A. Ahlsson, D. Atar, B. Casadei, et al., "2016 ESC guidelines for the management of atrial fibrillation developed in collaboration with EACTS," Rev Esp Cardiol (Engl Ed), vol. 70, p. 50, Jan 2017.

[4] S. Asgari, M. Bergsneider, and X. Hu, "A robust approach toward recognizing valid arterial-blood-pressure pulses," IEEE Trans Inf Technol Biomed, vol. 14, pp. 166-72, Jan 2010.

[5] S. Asgari, P. Xu, M. Bergsneider, and X. Hu, "A subspace decomposition approach toward recognizing valid pulsatile signals," Physiological Measurement, vol. 30, pp. 12111225, Nov 2009.

[6] W. Zong, T. Heldt, G. B. Moody, and R. G. Mark, "An opensource algorithm to detect onset of arterial blood pressure pulses," Computers in Cardiology 2003, Vol 30, vol. 30, pp. 259-262, 2003.

[7] A. Laurin and J. Joachim. (2017). Blood pressure waveform annotation. Available: https://github.com/jaj42/BP annotate

[8] J. Pan and W. J. Tompkins, "A real-time QRS detection algorithm," IEEE Trans Biomed Eng, vol. 32, pp. 230-6, Mar 1985.

[9] F. Shaffer and J. P. Ginsberg, "An Overview of Heart Rate Variability Metrics and Norms," Front Public Health, vol. 5, p. 258, 2017.

[10] J. S. Richman and J. R. Moorman, "Physiological time-series analysis using approximate entropy and sample entropy," American Journal of Physiology-Heart and Circulatory Physiology, vol. 278, pp. H2039-H2049, Jun 2000.

[11] G. Lu, F. Yang, J. A. Taylor, and J. F. Stein, "A comparison of photoplethysmography and ECG recording to analyse heart rate variability in healthy subjects," J Med Eng Technol, vol. 33, pp. 634-41, 2009.

[12] J. Claes, R. Buys, A. Avila, D. Finlay, A. Kennedy, D. Guldenring, et al., "Validity of heart rate measurements by the Garmin Forerunner 225 at different walking intensities," J Med Eng Technol, vol. 41, pp. 480-485, Aug 2017.

[13] S. E. Stahl, H. S. An, D. M. Dinkel, J. M. Noble, and J. M. Lee, "How accurate are the wrist-based heart rate monitors during walking and running activities? Are they accurate enough?," BMJ Open Sport Exerc Med, vol. 2, p. e000106, 2016.

Address for correspondence:

Kais Gadhoumi

Department of Physiological Nursing, School of Nursing

University of California San Francisco

2 Koret Way, San Francisco, CA, 94143, USA

kais.gadhoumi@ucsf.edu 